\title{
The Intermediate Surface Diffusion Flow on Spheres
}

Joachim ESCHER

Institute for Applied Mathematics, University of Hannover, D-30167 Hannover, Germany

E-mail: escher@ifam.uni-hannover.de

This paper is part of the

Proceedings of the Öresund Symposium on Partial Differential Equations;

Lund University, May 23-25, 2002

\begin{abstract}
It is shown that solutions to the intermediate surface diffusion flow are real analytic in space and time, provided the initial surface is real diffeomorphic to a Euclidean sphere.
\end{abstract}

\section{Introduction and main result}

Of concern is the following nonlocal geometric evolution problem. Given a compact closed orientable immersed $M$ hypersurface of $\mathbb{R}^{m+1}$, find a family $\mathcal{M}=\{M(t) ; t \geq 0\}$ of surfaces such that

$$
\begin{aligned}
& V(t)=\Delta_{M(t)}\left(\delta-\mu \Delta_{M(t)}\right)^{-1} H_{M(t)} \quad \text { on } \quad M(t) \quad \text { for } t>0, \\
& M(0)=M \quad \text { at } \quad t=0 .
\end{aligned}
$$

Here $V$ is the normal velocity of $\mathcal{M}$ and $\Delta_{M(t)}$ and $H_{M(t)}$ stand for the Laplace-Beltrami operator and the mean curvature of $M(t)$. Furthermore, $\delta$ and $\mu$ are positive constants. The evolution equation (1.1) does not depend on the local choice of orientation. However, if $M(t)$ encloses a domain $\Omega(t)$ (which is the physical relevant case), we always choose the orientation such that $V(t)$ is positive if $\Omega(t)$ grows and such that $H(t)$ is positive if $M(t)$ is convex with respect to $\Omega(t)$. We mention that in the plane case sometimes the opposite orientation is used.

Equation (1.1) is called intermediate surface diffusion flow and was first proposed by J W Cahn and J E Taylor [8] to describe a geometric growth law for a moving interface where surface diffusion is the only transport mechanism and the reduction of total surface energy is the only driving force for surface motion, cf. [8, 22]. In this framework, the constants $1 / \delta$ and $1 / \mu$ are called the diffusion coefficient and the mobility constant, respectively. Formally, as $\delta \rightarrow 0$ and $\mu \rightarrow 0$ the evolution law (1.1) connects the averaged mean curvarutre flow $V=\bar{H}_{M(t)}-H_{M(t)}$, where $\bar{H}_{M(t)}$ is the spatial average of $H_{M(t)}$, and the surface diffusion flow $V(t)=\Delta_{M(t)} H_{M(t)}$, see [8, 14]. This explains the name 
of (1.1). For the case $\mu=1$ and $\delta \rightarrow 0$ it is rigorously shown in [10] that the solutions to (1.1) converge in $C^{1,2+\alpha}$ to the corresponding solution of the averaged mean curvature flow. The limit $\mu \rightarrow 0$ is still open.

The first mathematical results for (1.1) was presented by C M Elliott and H Garcke [9]. They prove both global existence and stabilty results for plane curves when $M$ is close to a circle. These results were extended in [14] to the multi-dimensional case. More precisely, writing $h^{s}$ for the little Hölder spaces of order $s>0$, see Section 2, we have

Theorem 1.1. (i) Let $M$ be a surface of class $h^{2+\beta}$ with $\beta \in(0,1)$. Then the intermediate surface diffusion flow (1.1) possesses a unique classical solution $\mathcal{M}=\left\{M(t) ; t \in\left[0, t^{+}\right)\right\}$, where $t^{+}:=t^{+}(M)>0$ is the maximal existence time. The mapping $[t \mapsto M(t)]$ is smooth on $\left(0, t^{+}\right)$with respect to the $C^{\infty}$-topology and continuous on $\left[0, t^{+}\right)$with respect to the $h^{2+\beta}$-topolgy.

(ii) Let $S$ be a fixed Euclidean sphere and let $\mathcal{S}$ denote the set of all spheres which are sufficiently $C^{2+\beta}$-close to $\mathcal{S}$ Then $\mathcal{S}$ attracts at an exponential rate all solutions which are $C^{2+\beta}$-close to $\mathcal{S}$. In particular, all solutions starting from such a neighbourhood exists globally and converge exponentially fast to some sphere as $t \rightarrow \infty$. The convergence is in the $C^{k}$-topology for any fixed $k \in \mathbb{N}$.

The purpose of this paper is to extend the existence and uniqueness part of Theorem 1.1 to $h^{1+\alpha}$ initial data and to show that the solutions to (1.1) are actually real analytic, provided $M$ is chosen suitably. More precisely, we shall prove the following result:

Theorem 1.2. (i) Let $\Sigma$ be a smooth compact closed immersed oriented hypersurface of $\mathbb{R}^{m+1}$ and assume that $M$ is the graph over $\Sigma$ of a $h^{1+\alpha}$-function with $\alpha \in(1 / 2,1)$. Then (1.1) possesses a unique solution $\left\{M(t) ; t \in\left[0, t^{+}\right)\right\}$in

$$
C\left(\left[0, t^{+}\right), h^{1+\alpha}(\Sigma)\right) \cap C^{1}\left(\left[0, t^{+}\right), h^{\alpha-1}(\Sigma)\right) .
$$

The mapping $[(t, M) \mapsto M(t)]$ is a continuous semiflow on an open subset of $h^{1+\alpha}(\Sigma)$.

(ii) Assume that $\Sigma$ is real analytically diffeomorphic to the Euclidean sphere $\mathbb{S}^{m}$. Then

$$
\bigcup_{t \in\left(0, t^{+}\right)}(\{t\} \times M(t))
$$

is a real analytic manifold.

Theorem 1.2 is obtained by applying a recent result on the smoothing property of abstract fully nonlinear parabolic evolution equations on symmetric spaces, cf. [15]. We present this general framework in Section 4. It is based on the concept of continuous maximal regularity which will be introduced in Section 2. In Section 3 we provide a suitable parametrization of a tubular neighbourhood $\mathcal{R}$ of $\Sigma$ which allows to translate (1.1) in $\mathcal{R}$ into a nonlinear nonlocal parabolic evolution equation on the reference manifold $\Sigma$.

\section{Continuous maximal regularity}

In this section we briefly introduce the notion of maximal regularity in the sense of Da Prato-Grisvard. For this let $E_{0}$ and $E_{1}$ be Banach spaces such that $E_{1}$ is continuously injected and dense in $E_{0}$. Let $\mathcal{H}\left(E_{1}, E_{0}\right)$ denote the subset of all $A \in \mathcal{L}\left(E_{1}, E_{0}\right)$ 
such that $-A$, considered as a, in general, unbounded operator in $E_{0}$, generates a strongly continuous analytic semigroup on $E_{0}$. Let $B \subset E_{1}$ be open and assume that

$$
P \in C^{\omega}\left(B, E_{0}\right) \quad \text { with } \quad \partial P(v) \in \mathcal{H}\left(E_{1}, E_{0}\right), \quad v \in B
$$

where $\partial P(v) h:=\left.\frac{d}{d \varepsilon} P(v+\varepsilon h)\right|_{\varepsilon=0}$ denotes the Fréchet derivative of $P$ at $v \in B$.

Given $T>0$, set

$$
\mathbb{E}_{0}:=C\left([0, T], E_{0}\right), \quad \mathbb{E}_{1}:=C\left([0, T], E_{1}\right) \cap C^{1}\left([0, T], E_{0}\right),
$$

and let $\gamma: \mathbb{E}_{0} \rightarrow E_{0}, u \mapsto u(0)$ denote the trace operator in $\mathbb{E}_{0}$. We assume that $\left(\mathbb{E}_{0}, \mathbb{E}_{1}\right)$ is a pair of maximal regularity for $\partial P(v)$, this means we assume that

$$
\left(\frac{d}{d t}+\partial P(v), \gamma\right) \in \operatorname{Isom}\left(\mathbb{E}_{1}, \mathbb{E}_{0} \times E_{1}\right), \quad v \in B .
$$

We are now ready to formulate the following existence and uniqueness result:

Theorem 2.1. Assume that (2.1) and (2.2) hold true. Then, given any $u_{0} \in B$ and $f \in C^{\omega}\left(\mathbb{R}_{+}, E_{0}\right)$, there exist $t^{+}:=t^{+}\left(u_{0}\right)>0$ and a unique maximal solution

$$
u:=u\left(\cdot, u_{0}\right) \in C\left(\left[0, t^{+}\right), B\right) \cap C^{1}\left(\left[0, t^{+}\right), E_{0}\right)
$$

of the initial value problem

$$
\frac{d}{d t} u+P(u)=f, \quad u(0)=u_{0} .
$$

Remarks 2.2. a) Theorem 2.1 essentially goes back to Da Prato and Grisvard [7]. For some refinements and generalizations see also [4].

b) Observe that assumption (2.2) and Theorem 2.1 coincide in the linear case, i.e., if $B=E_{1}$ and $P \in \mathcal{L}\left(E_{1}, E_{0}\right)$. Nevertheless, it is not at all clear whether or not property (2.2) can be verified if $E_{1} \neq E_{0}$. In fact, it follows from a result of Baillon [6] that, in case $E_{1} \neq$ $E_{0}$, property (2.3) can only be expected if $E_{0}$ contains an isomorphic copy of the sequence space $c_{0}$. In particular, (2.3) will never be true in reflexive Banach spaces. However, in [7] the continuous interpolation functor $(\cdot, \cdot)_{\theta, \infty}^{0}$ was introduced, an interpolation method producing non-reflexive Banach spaces for which condition (2.2) can be verified.

c) Let us briefly introduce an important scale of Banach spaces, which may be realized as continuous interpolation spaces. Given $s \in \mathbb{R}$, define the little Hölder to be

$$
b u c^{s}\left(\mathbb{R}^{m}\right):=\text { closure of } B U C^{\infty}\left(\mathbb{R}^{m}\right) \text { in } B_{\infty, \infty}^{s}\left(\mathbb{R}^{m}\right) \text {, }
$$

where $B_{\infty, \infty}^{s}\left(\mathbb{R}^{m}\right)$ stands for the Besov spaces as defined in [23]. Note that the spaces $B_{\infty, \infty}^{s}\left(\mathbb{R}^{m}\right)$ coincides with the ususal Hölder spaces $B U C^{s}\left(\mathbb{R}^{m}\right)$, provided $s>0$ is not an integer, see Theorem 2.5.7 and Remark 2.2.2.3 in [23]. Then it is shown in [19], Theorem 1.2.17 that

$$
\left(B U C\left(\mathbb{R}^{m}\right), B U C^{n}\left(\mathbb{R}^{m}\right)\right)_{\theta, \infty}^{0}=b u c^{\theta n}\left(\mathbb{R}^{m}\right)
$$

for all $n \in \mathbb{N}$ and $\theta \in(0,1)$ such that $\theta n \notin \mathbb{N}$. 
d) Assume that $M$ is a smooth Riemannian manifold with bounded curvature and positive radius of injectivity. Then Lemma 2.26 in [5] ensures the existence of a uniformly locally finite covering of geodesic balls $M\left(p_{j}, \delta\right)$ with $p_{j} \in M, j \in \mathbb{N}$ and $\delta>0$. As before the spaces $b u c^{s}(M)$ are defined to be the closure of $B U C^{\infty}(M)$ in $B U C^{s}(M)$. Again we have that

$$
\left(B U C(M), B U C^{n}(M)\right)_{\theta, \infty}^{0}=b u c^{\theta n}(M)
$$

for all $n \in \mathbb{N}$ and $\theta \in(0,1)$ such that $\theta n \notin \mathbb{N}$, cf. the proof of Corollary 1.2.19 in [19]. For simplicity we write $h^{s}(M)=b u c^{s}(M)$ for $s \in \mathbb{R}$ if $M$ is compact.

e) A further scale of Banach spaces for which maximal regularity can be verified are the so called little Nikol'skii spaces. They can be realized as continuous interpolation spaces of Bessel potential spaces, cf. [7], Section 6 and [21], Section 6.

f) Consider again the "linear" case $B=E_{1}$ and $P \in \mathcal{L}\left(E_{1}, E_{0}\right)$ and suppose in addition that $f \equiv 0$. Then problem (2.4) has for each $u_{0} \in E_{1}$ a unique solution in the class $\mathbb{E}_{1}$ (for any $T>0$, of course), provided $-P$ generates a strongly continuous semigroup, which does not need to be analytic. However, it is shown in [7] that the semigroup is automatically analytic if condition (2.2) is supposed to hold, see also Proposition III.3.1.1 in [2].

g) A well-known characterization of generators of analytic semigroups yields that $A \in$ $\mathcal{L}\left(E_{1}, E_{0}\right)$ belongs to $\mathcal{H}\left(E_{1}, E_{0}\right)$ if there are positive constants $\kappa$ and $\omega$ such that $[\operatorname{Re} \lambda \geq$ $\omega] \subset \rho(-A)$ and

$$
|\lambda|\left\|(\lambda+A)^{-1}\right\|_{\mathcal{L}\left(E_{0}\right)} \leq \kappa, \quad \operatorname{Re} \lambda \geq \omega .
$$

h) Finally, we mention that Theorem 2.1 remains true under a much weaker regularity assumption for $P$. Indeed, it suffices to assume that $P$ is continuously Fréchet differentiable. Under these regularity assumption it can also be shown that the mapping

$$
\bigcup_{x \in B}\left(\left[0, t^{+}(x)\right) \times\{x\}\right) \rightarrow B, \quad(t, x) \mapsto u(t, x)
$$

is a semiflow on $B$, provided $f$ does not depend on $t$. However, since we are looking for possible smoothing properties of solutions, we presuppose analyticity of $P$ from the very beginning.

\section{Parametrization}

We parametrize (1.1) in a neighbourhood of an analytic compact closed immersed oriented hypersurface $\Sigma$ in $\mathbb{R}^{m+1}$. To make this precise, let $\nu$ denote the unit outer normal field on $\Sigma$. Moreover, given $a>0$, choose a localization system $\left\{\left(U_{l}, \varphi_{l}\right) ; l=1, \ldots, n\right\}$ for $\Sigma$ such that $\Sigma=\cup_{l=1}^{n} U_{l}$ and

$$
\varphi_{l}:(-a, a)^{m} \rightarrow U_{l}, \quad l \in\{1, \ldots, n\},
$$

is an analytic parametrization of $U_{l}$. Shrinking $a>0$ if necessary, we may assume that

$$
X_{l}: U_{l} \times(-a, a) \rightarrow \mathbb{R}^{m+1}, \quad X_{l}(s, r):=s+r \nu(s)
$$


is a smooth diffeomorphism onto its image $\mathcal{R}_{l}:=\operatorname{im}\left(X_{l}\right)$, i.e.

$$
X_{l} \in \operatorname{Diff}^{\omega}\left(U_{l} \times(-a, a), \mathcal{R}_{l}\right) .
$$

The inverse of $X_{l}$ can be decomposed in the following way. Writing $S_{l} \in C^{\omega}\left(\mathcal{R}_{l}, U_{l}\right)$ and $\Lambda_{l} \in C^{\omega}\left(\mathcal{R}_{l},(-a, a)\right)$ for the metric projection of $\mathcal{R}_{l}$ onto $U_{l}$ and for the signed distance function with respect to $U_{l}$, respectively, we have $X_{l}^{-1}=\left(S_{l}, \Lambda_{l}\right)$. In particular, observe that $\mathcal{R}:=\cup_{l=1}^{n} \mathcal{R}_{l}$ consists of those points in $\mathbb{R}^{m+1}$ with distance less than $a$ to $\Sigma$.

We now fix $\alpha>1 / 2$, let

$$
W(\Sigma):=W_{a}(\Sigma):=\left\{\rho \in h^{1+\alpha}(\Sigma) ;\|\rho\|_{C^{1}(\Sigma)}<a / 2\right\}
$$

and define

$$
M_{\rho}:=\bigcup_{l=1}^{n}\left\{X_{l}(s, \rho(s)) ; s \in U_{l}\right\}
$$

for $\rho \in W(\Sigma)$. Then $M_{\rho}$ is a compact closed oriented immersed hypersurface in $\mathbb{R}^{m+1}$ of class $h^{1+\alpha}$, which can be seen as a graph in normal direction over $\Sigma$. Of course, $\rho$ measures the signed distance of $\Sigma$ to $M_{\rho}$. For convenience let us also introduce the mapping

$$
\theta_{\rho}: \Sigma \rightarrow M_{\rho}, \quad s \mapsto X_{l}(s, \rho(s)) \quad \text { for } \quad s \in U_{l}
$$

Then $\theta_{\rho}$ is a well-defined global diffeomorphism of class $h^{1+\alpha}$ from $\Sigma$ onto $M_{\rho}$. By means of this diffeomorphism we can pull back the Euclidean metric on $M_{\rho}$ to $\Sigma$, producing in that way a Riemannian manifold which we denote in the following by $\Sigma(\rho)$. We now consider a family of hypersurfaces in $\mathcal{R}$. More precisely, let $T>0$ be given, and define $I:=[0, T]$, as well as

$$
W\left(\Sigma_{T}\right):=W_{a}\left(\Sigma_{T}\right):=\left\{\rho \in C\left(I, h^{1+\alpha}(\Sigma)\right) ;\|\rho\|_{C\left(I, C^{1}(\Sigma)\right)}<a / 2\right\} .
$$

Then, given $\rho \in W\left(\Sigma_{T}\right)$, we transform the evolution equation (1.1) for the family $\left\{M_{\rho(t)}\right.$; $t \in[0, T]\}$ into an evolution equation on $\Sigma$. For this we first calculate the normal velocity of $\left[t \mapsto M_{\rho(t)}\right]$. We have, cf. [11],

$$
V(t, s)=\partial_{t} \rho(t, s) /\left|\nabla_{x} \Phi_{\rho}(x, t)\right|_{x=\theta_{\rho(t)}(s)} \quad \text { for } \quad(t, s) \in I \times \Sigma,
$$

where we used the function

$$
\Phi_{\rho}: \mathcal{R} \times[0, T] \rightarrow \mathbb{R}, \quad(x, t) \mapsto \Lambda(x)-\rho(t, S(x))
$$

to represent $M_{\rho(t)}$ as the 0 -level set of $\Phi_{\rho}(\cdot, t)$, i.e. $M_{\rho(t)}=\Phi^{-1}(\cdot, t)(0)$. To shorten our notation, let

$$
L(\rho)(t, s):=\left|\nabla_{x} \Phi_{\rho}(x, t)\right|_{x=\theta_{\rho(t)}(s)}, \quad(t, s) \in I \times \Sigma .
$$

Moreover, we write $K(\rho):=\theta_{\rho}^{*} H$ and $\Delta_{\rho}$ for the mean curvature and Laplace-Beltrami operator of $\Sigma(\rho)$, respectively. Here, $\theta_{\rho}^{*}$ denotes the pull-back operator induced by the 
diffeomorphism $\theta_{\rho}$, i.e. $\theta_{\rho}^{*} f=f \circ \theta_{\rho}$ for $f \in C\left(M_{\rho}\right)$. This means in particular that we have

$$
\theta_{\rho}^{*} \Delta_{M_{\rho}}=\Delta_{\rho} \theta_{\rho}^{*}
$$

Given $\rho_{0} \in W_{a}(\Sigma)$, consider now the following nonlinear nonlocal partial differential equation

$$
\frac{d \rho}{d t}=L(\rho) \Delta_{\rho}\left(\delta-\mu \Delta_{\rho}\right)^{-1} K(\rho) \quad \text { in } \quad I \times \Sigma, \quad \rho(0)=\rho_{0} \quad \text { on } \quad \Sigma .
$$

In order to treat (3.1) in the framework of Theorem 2.1, we set

$$
P(\rho):=L(\rho) \Delta_{\rho}\left(\delta-\mu \Delta_{\rho}\right)^{-1} K(\rho) \quad \text { for } \quad \rho \in W(\Sigma) \cap h^{2+\alpha}(\Sigma) .
$$

Our first result in this section shows that $P$ can be extended to an analytic mapping with values in $h^{\alpha-1}(\Sigma)$.

Lemma 3.1. There exists an extension of $P$, again denoted by $P$, which belongs to the space $P \in C^{\omega}\left(W(\Sigma), h^{\alpha-1}(\Sigma)\right)$.

Proof. (i) We first express the terms $L(\rho), K(\rho)$, and $\Delta_{\rho}$ in local coordinates. To make this precise, let

$$
\hat{\rho}_{l}(s):=\rho\left(\varphi_{l}(s)\right), \quad \hat{X}_{l}(s, r):=X_{l}\left(\varphi_{l}(s), r\right), \quad(s, r) \in(-a, a)^{m+1},
$$

be the local representations of $\rho_{l}$ and $X_{l}$ with respect to $U_{l}$. In the following we do not always distinguish between $\rho_{l}, X_{l}$ and their local representations $\hat{\rho}_{l}, \hat{X}_{l}$, as well as between local coordinates $s \in(-a, a)^{m}$ and the corresponding points $\varphi_{l}(s)$ on $U_{l}$. Moreover, we suppress the index $l \in\{1, \ldots, n\}$ if no confusion seems likely. Given $\rho \in W(\Sigma)$, define

$$
w_{j k}(\rho)(s):=\left.\left(\partial_{j} X \mid \partial_{k} X\right)\right|_{(s, \rho(s))}, \quad s \in(-a, a)^{m},
$$

for $j, k \in\{1, \ldots, m\}$, where $(\cdot \mid \cdot)$ stands for the Euclidean metric in $\mathbb{R}^{m+1}$ and $\partial_{j}$ denotes the partial derivative with respect to the $j$-th variable of $s$. Since $\rho$ belongs to $W(\Sigma)$, the matrix $\left[w_{j k}(\rho)\right]$ is invertible and we write $w^{j k}(\rho)$ for the entries of its inverse. Then we have

$$
L(\rho)=\sqrt{1+w^{j k}(\rho) \partial_{j} \rho \partial_{k} \rho}
$$

cf. (2.3) in [13]. In (3.2) and in what follows we use summation convention over repeated indices. Moreover, we write

$$
\Gamma_{j k}^{i}(\rho):=\left.\frac{1}{2} w^{i l}(\rho)\left(\partial_{k}\left(\partial_{l} X \mid \partial_{j} X\right)-\partial_{l}\left(\partial_{j} X \mid \partial_{k} X\right)+\partial_{j}\left(\partial_{k} X \mid \partial_{l} X\right)\right)\right|_{(\cdot, \rho)},
$$

for the corresponding Christoffel symbols. Then Lemma 2.1 in [13] shows that $K(\rho)$ carries a quasi-linear structure, i.e. given $\rho \in W(\Sigma)$, there are

$$
K_{1}(\rho) \in \mathcal{L}\left(h^{2+\alpha}(\Sigma), h^{\alpha}(\Sigma)\right) \quad \text { and } \quad K_{2}(\rho) \in h^{\alpha}(\Sigma)
$$


such that

$$
K(\rho)=K_{1}(\rho) \rho+K_{2}(\rho) \quad \text { for } \quad \rho \in W(\Sigma) \cap h^{2+\alpha}(\Sigma) .
$$

In the chosen local coordinates these mappings are represented as:

$$
\begin{aligned}
K_{1}(\rho)= & \frac{1}{m L(\rho)^{3}}\left[\left\{-L(\rho)^{2} w^{j k}(\rho)+w^{j l}(\rho) w^{k n}(\rho) \partial_{l} \rho \partial_{n} \rho\right\} \partial_{j} \partial_{k}\right. \\
& +\left\{L(\rho)^{2} w^{j k}(\rho) \Gamma_{j k}^{i}(\rho)+w^{j l}(\rho) w^{k i}(\rho) \Gamma_{j k}^{m+1}(\rho) \partial_{l} \rho\right. \\
& \left.\left.+2 w^{k n}(\rho) \Gamma_{(m+1) k}^{i}(\rho) \partial_{n} \rho-w^{j l}(\rho) w^{k n}(\rho) \Gamma_{j k}^{i}(\rho) \partial_{l} \rho \partial_{n} \rho\right\} \partial_{i}\right]
\end{aligned}
$$

and

$$
K_{2}(\rho)=-\frac{1}{m L(\rho)} w^{j k}(\rho) \Gamma_{j k}^{m+1}(\rho) .
$$

In order to express $\Delta_{\rho}$ in local coordinates, let $\eta$ be the Euclidean metric and write $\sigma(\rho):=\theta_{\rho}^{*} \eta$ for the Riemannian metric on $\Sigma$ induced by the diffeomorphism $\theta_{\rho}$. This means that, using the above introduced notation, we have $\Sigma(\rho)=(\Sigma, \sigma(\rho))$. Let further $\sigma_{j k}(\rho)$ denote the components of $\sigma(\rho)$ in local coordinates and write $\sigma^{j k}(\rho)$ for the components of the inverse of $\left[\sigma_{j k}(\rho)\right]$. Using again summation convention over repeated indices, the Christoffel symbols of $\sigma(\rho)$ in the chosen coordinates are given by

$$
\gamma_{j k}^{l}(\rho)=\frac{\sigma^{l n}(\rho)}{2}\left[\frac{\partial \sigma_{k n}(\rho)}{\partial s^{j}}+\frac{\partial \sigma_{j n}(\rho)}{\partial s^{k}}-\frac{\partial \sigma_{j k}(\rho)}{\partial s^{n}}\right],
$$

and we have

$$
\Delta_{\rho}=\sigma^{j k}(\rho)\left[\frac{\partial^{2}}{\partial s^{j} \partial s^{k}}-\gamma_{j k}^{l}(\rho) \frac{\partial}{\partial s^{l}}\right], \quad \rho \in W(\Sigma) \cap h^{2+\alpha}(\Sigma),
$$

cf. the proof of Lemma 2.1 in [13].

(ii) It follows from $\left[11\right.$, p. 1037] that $w_{j k}(\rho)$ is a quadratic polynomial in $\rho$. Moreover, we have that

$$
\left[\rho \mapsto \theta_{\rho}-i d_{\Sigma}\right] \in \mathcal{L}\left(h^{1+\alpha}(\Sigma), h^{1+\alpha}\left(\Sigma, \mathbb{R}^{m+1}\right)\right)
$$

This obviously implies that

$$
w_{j k} \in C^{\omega}\left(W(\Sigma), h^{1+\alpha}(\Sigma)\right), \quad \sigma_{j k} \in C^{\omega}\left(W(\Sigma), h^{\alpha}(\Sigma)\right),
$$

and consequently:

$$
w^{j k}, \Gamma_{j k}^{i} \in C^{\omega}\left(W(\Sigma), h^{1+\alpha}(\Sigma)\right), \quad \sigma^{j k} \in C^{\omega}\left(W(\Sigma), h^{\alpha}(\Sigma)\right) .
$$

(iii) Combining (3.9) and (3.2), we see that

$$
L \in C^{\omega}\left(W(\Sigma), h^{\alpha}(\Sigma)\right) .
$$


Recall that we have assumed that $\alpha>1 / 2$. Hence $\alpha>\max \{1-\alpha, \alpha-1\}$, and we conlcude from [23, Theorem 2.8.2] and a localization argument that

$$
h^{\alpha}(\Sigma) \times h^{\alpha-1}(\Sigma) \rightarrow h^{\alpha-1}(\Sigma), \quad(f, g) \mapsto f g
$$

is continuous and bilinear. Using this and Theorem 2.3.8 in [23], it follows from (3.5), (3.6), and (3.9) that

$$
[\rho \mapsto K(\rho)] \in C^{\omega}\left(W(\Sigma), h^{\alpha-1}(\Sigma)\right) .
$$

(iv) Let $\rho_{0} \in W(\Sigma)$ be given and choose $\varepsilon>0$ such that

$$
B_{0}:=\mathbb{B}_{h^{1+\alpha}(\Sigma)}\left(\rho_{0}, \varepsilon_{0}\right) \subset W(\Sigma) .
$$

For simplicity we set $X_{0}:=h^{\alpha-1}(\Sigma)$ and $X_{1}:=h^{1+\alpha}(\Sigma)$, and denote the $X_{0}$-realization of $\delta-\mu \Delta_{\rho}$ by $A(\rho)$. Shrinking $\varepsilon_{0}>0$, it follows from Theorem 4.2 in [3] and a localization argument that

$$
A(\rho) \in \mathcal{H}\left(X_{1}, X_{0}\right), \quad \rho \in B_{0} .
$$

Moreover, we conclude from Theorem 7.4.3 and Remark 7.2.5.1 in [24] that $\mathbb{R}_{+}$belongs to the resolvent set $\operatorname{res}(A(\rho))$ of $A(\rho)$ for all $\rho \in B_{0}$. Observing (3.7), we infer from (3.13) that

$$
\left[\rho \mapsto A(\rho) A(0)^{-1}\right],\left[\rho \mapsto \Delta_{\rho} A(0)^{-1}\right] \in C^{\omega}\left(B_{0}, \mathcal{L}\left(X_{0}\right)\right) .
$$

The first assertion in (3.14) yields

$$
\left[\rho \mapsto A(0) A(\rho)^{-1}\right] \in C^{\omega}\left(B_{0}, \mathcal{L}\left(X_{0}\right)\right),
$$

since $\left[B \mapsto B^{-1}\right] \in C^{\omega}\left(\mathcal{L}\left(X_{0}\right)\right)$, see Theorem VIII.7 in [25]. From the second assertion in (3.14) and (3.15) we thus get

$$
\left[\rho \mapsto \Delta_{\rho} A(\rho)^{-1}\right] \in C^{\omega}\left(B_{0}, \mathcal{L}\left(X_{0}\right)\right) .
$$

Since pointwise multiplication in $h^{\alpha}(\Sigma)$ is bilinear and continuous, it remains to combine (3.10), (3.12), and (3.16) to complete the argumentation.

Lemma 3.2. Given $\rho \in W(\Sigma)$, we have

$$
\partial P(\rho) \in \mathcal{H}\left(h^{1+\alpha}(\Sigma), h^{\alpha-1}(\Sigma)\right) .
$$

Proof. Let $\rho \in W(\Sigma)$ be given. Then

$$
\begin{aligned}
P(\rho) & =\frac{1}{\mu} L(\rho) K(\rho)-\frac{\delta}{\mu} L(\rho)\left(\delta-\mu \Delta_{\rho}\right)^{-1} K(\rho) \\
& =\frac{1}{\mu} L(\rho) K_{1}(\rho) \rho+\frac{1}{\mu} L(\rho)\left[K_{2}(\rho)-\delta\left(\delta-\Delta_{\rho}\right)^{-1} K(\rho)\right]
\end{aligned}
$$

Observing (3.5), (3.6), (3.7), and (3.10) we find that there is a

$$
R \in C^{\omega}\left(W(\Sigma), \mathcal{L}\left(h^{1+\alpha}(\Sigma), h^{\alpha}(\Sigma)\right)\right.
$$


such that

$$
\partial P(\rho) h=\frac{1}{\mu} L(\rho) K_{1}(\rho) h+R(\rho) h, \quad(\rho, h) \in W(\Sigma) \times h^{1+\alpha}(\Sigma) .
$$

It follows from Lemma 3.2 in [12] that $K_{1}(\rho)$ is a strongly elliptic differential operator with coefficient belonging to $h^{\alpha}(\Sigma)$. Moreover, $L(\rho) \in h^{\alpha}(\Sigma)$ is positive. Thus we infer from Theorem 4.2 in [3] that $K_{1}(\rho) \in \mathcal{H}\left(h^{1+\alpha}(\Sigma), h^{\alpha-1}(\Sigma)\right)$. Combining this with (3.17), a well-known perturbation result for generators of analytic semigroups shows that $\partial P(\rho) \in$ $\mathcal{H}\left(h^{1+\alpha}(\Sigma), h^{\alpha-1}(\Sigma)\right)$.

Corollary 3.3. Let $E_{j}:=h^{\alpha-1+2 j}(\Sigma)$ for $j=0,1$ and $B:=W(\Sigma)$. Then the operator $P$ satisfies the hyptheses (2.1) and (2.2).

Proof. Let $\beta \in(1 / 2, \alpha)$ be fixed. Then, given $\rho \in B$, the very same arguments as in Lemma 3.2 ensure that $\partial P(\rho) \in \mathcal{H}\left(h^{1+\beta}\left(\Sigma^{m}\right), h^{\beta-1}\left(\Sigma^{m}\right)\right)$. Since the spaces $h^{s}\left(\Sigma^{m}\right)$ are stable under continuous interpolation and since the maximal $E_{0}$-realization of $\partial P(\rho) \in$ $\mathcal{L}\left(h^{1+\beta}\left(\Sigma^{m}\right), h^{\beta-1}\left(\Sigma^{m}\right)\right)$ obviously coincides with $\partial P(\rho) \in \mathcal{L}\left(E_{1}, E_{0}\right)$, Theorem 2.3 in [21] implies that $P$ satisfies assumption (2.2) as well.

Corollary 3.4. Let $\rho_{0} \in W(\Sigma)$ be given and let $M$ be the graph of $\rho_{0}$ in normal direction. Then there is a $T=T(M)>0$ such that (1.1) possesses a unique solution $\left\{M_{\rho(t)} ; t \in\right.$ $[0, T)\}$ with

$$
\rho \in C\left([0, T), h^{1+\alpha}(\Sigma)\right) \cap C^{1}\left([0, T), h^{\alpha-1}(\Sigma)\right) .
$$

Proof. This follows from Corollary 3.3 and Theorem 2.1.

\section{The smoothing property}

Let $\Sigma$ be an analytic closed Riemannian manifold of dimension $m$ and assume that $E_{0}$ and $E_{1}$ are Banach spaces of functions over $\Sigma$. More precisely, assume that $E_{1}$ is dense in $E_{0}$ and that

$$
E_{1} \hookrightarrow B U C(\Sigma), \quad E_{1} \hookrightarrow E_{0} \hookrightarrow \mathcal{D}^{\prime}(\Sigma)
$$

where $\mathcal{D}^{\prime}(\Sigma)$ stands for the space of all distributions on $\Sigma$. Throughout this section we presuppose (2.1) and (2.2) and we let $u$ denote the solution of $(2.4)$ on $\left[0, t^{+}\right)$, where $u_{0} \in B$ is given and where we assume for simplicity that $f \equiv 0$. Moreover, we set $\hat{u}(t, q):=u(t)(q)$ for $(t, q) \in\left[0, t^{+}\right) \times \Sigma$. Our goal is to show that $u$ enjoys a smoothing property. Hence, subdividing the interval of existence and using the semiflow property of $u$, see Remark $2.2(\mathrm{~h})$, we may assume without loss of generality that $t^{+} \leq 1$. Further, we fix $T \in\left(0, t^{+}\right)$and set $I:=[0, T]$.

From Theorem 2.1 we know that $u$ belongs to $C(I, B) \cap C^{1}\left(I, E_{0}\right)$. Since we are dealing with nonlinear equations, including fully nonlinear partial differential equations involving nonlocal terms too, there is no reason to expect $u$ to have any further regularity, like

$$
u \in C^{\alpha}\left(I \backslash\{0\}, E_{1}\right) \quad \text { or } \quad u \in C\left(I \backslash\{0\},\left(E_{1}, E_{2}\right)_{\alpha}\right),
$$


where $E_{2}$ stands for the domain of definition of $\left[\partial P\left(u_{0}\right)\right]^{2}$, equipped with the corresponding graph norm and where $(\cdot, \cdot)_{\alpha}$ denotes a suitable interpolation method. However, it turns out that there is actually a strong smoothing property for solutions of problem (2.4), provided we impose suitable symmetry properties for the manifold $\Sigma$ as well as for the nonlinear operator $P$.

It should be remarked that if $P$ carries a quasilinear structure in the sense that Theorem 12.1 in [1] is applicable it can be shown that the corresponding solutions do in fact possess a smoothing property in the sense of (4.1) without any geometrical condition on $\Sigma$ or on $P$.

Concerning the manifold $\Sigma$ we shall assume that it is analytically diffeomorphic to a globally Riemannian symmetric space. More precisely, we assume that

there exists a globally symmetric

Riemannian space $M$ and a $\Phi \in \operatorname{Diff}^{\omega}(M, \Sigma)$.

Recall that a Riemannian manifold $M$ is called a globally symmetric space if it is connected and if for each $p \in M$ there is a involutive isometry $\sigma_{p}: M \rightarrow M$ such that $p$ is an isolated fixed point of $\sigma_{p}$. Observe that $\sigma_{p}$ reverses in particular geodesics passing through the point $p$. This implies that $M$ is complete and, by the Hopf-Rinow theorem, that the group $I(M)$ of all isometries acts transitively on $M$. Observe further that $\left(\mathrm{A}_{2}\right)$ implies that $\left(\Sigma, \Phi_{*} g\right)$ is a globally symmetric Riemannian space, where $g$ denotes the metric of $M$. However, in view of applications, we prefer to keep the original metric on $\Sigma$.

Let now $\Phi^{*}$ and $\Phi_{*}$ denote the pull back and push forward operator induced by $\Phi$. This means that, given $v \in \mathcal{D}(\Sigma)$ and $w \in \mathcal{D}(M)$, we have

$$
\Phi^{*} v:=v \circ \Phi \quad \text { and } \quad \Phi_{*} w:=w \circ \Phi^{-1},
$$

where, of course, $\mathcal{D}(\Sigma)$ stands for the space of all test functions over $\Sigma$. It follows from Theorem 2.2.26 and Corollary 2.2.21 in [17] that $M$ has bounded curvature and a positive radius of injectivity $\delta>0$. Hence Lemma 2.26 in [5] ensures that there exists a uniformly locally finite covering of geodesic balls $M\left(p_{j}, \delta\right)$ on $M$ and a smooth partition of unity $\left\{\pi_{j} ; j \in \mathbb{N}\right\}$ subordinated to $\left\{M\left(p_{j}, \delta\right) ; j \in \mathbb{N}\right\}$. Using this partion of unity it is not difficult to verify by duality that

$$
\Phi^{*} \in \operatorname{Isom}\left(\mathcal{D}^{\prime}(\Sigma), \mathcal{D}^{\prime}(M)\right), \quad \Phi_{*} \in \operatorname{Isom}\left(\mathcal{D}^{\prime}(M), \mathcal{D}^{\prime}(\Sigma)\right) \quad \text { with } \quad\left[\Phi^{*}\right]^{-1}=\Phi_{*} .
$$

For $j=0,1$, let now

$$
F_{j}:=\left\{\Phi^{*} v ; v \in E_{j}\right\}, \quad\|w\|_{F_{j}}:=\left\|\Phi_{*} w\right\|_{E_{j}}, \quad w \in F_{j} .
$$

Then $F_{j}:=\left(F_{j} ;\|\cdot\|_{F_{j}}\right)$ are well-defined Banach spaces such that $F_{1}$ is continuously injected and dense in $F_{0}$. Moreover, we have $F_{0} \subset \mathcal{D}^{\prime}(M)$ and $F_{1} \subset B U C(M)$. We next introduce

$$
Q(w, \Phi):=\Phi^{*} P\left(\Phi_{*} w\right), \quad v \in D
$$

where $D:=\left\{\Phi^{*} v ; v \in B\right\}$. Of course,

$$
D \text { is open in } F_{1} \text { and } Q(\cdot, \Phi) \in C^{\omega}\left(D, F_{0}\right) \text {, }
$$


because of the fact that $\Phi^{*}: E_{j} \rightarrow F_{j}$ is an isometric isomorphism. We write $\partial_{1} Q(w, \Phi) \in$ $\mathcal{L}\left(F_{1}, F_{0}\right)$ for the Fréchet derivative of $Q(\cdot, \Phi)$. Further, we need the spaces

$$
\mathbb{F}_{0}:=C\left(I, F_{0}\right), \quad \mathbb{F}_{1}:=C\left(I, F_{1}\right) \cap C^{1}\left(I, F_{0}\right)
$$

The pull back and push forward operator induced by $\Phi$ on $\mathbb{E}_{0}$ and $\mathbb{F}_{0}$ are defined pointwise with respect to $t \in I$, i.e., given $v \in \mathbb{E}_{0}$ and $w \in \mathbb{F}_{0}$, let

$$
\Phi^{*} v: I \rightarrow F_{0}, \quad t \mapsto \Phi^{*} v(t), \quad \Phi_{*} w: I \rightarrow E_{0}, \quad t \mapsto \Phi_{*} w(t) .
$$

Of course we do also not distinguish notationally between $\Phi^{*}$ and $\Phi_{*}$ and restrictions of these operators to linear subspaces of $\mathbb{E}_{0}$ and $\mathbb{F}_{0}$, respectively.

Lemma 4.1. The following assertions hold true:

(i) $\Phi^{*} \in \operatorname{Isom}\left(\mathbb{E}_{j}, \mathbb{F}_{j}\right), \Phi_{*} \in \operatorname{Isom}\left(\mathbb{F}_{j}, \mathbb{E}_{j}\right)$ and $\left[\Phi^{*}\right]^{-1}=\Phi_{*}$ for $j=0,1$.

(ii) $\left(\mathbb{F}_{1}, \mathbb{F}_{0}\right)$ is a pair of maximal regularity for $\partial_{1} Q(\cdot, \Phi)$, i.e.,

$$
\left(\frac{d}{d t}+\partial_{1} Q(w, \Phi), \gamma\right) \in \operatorname{Isom}\left(\mathbb{F}_{1}, \mathbb{F}_{0} \times F_{1}\right), \quad w \in D
$$

Proof. The first assertion follows from the construction of the spaces $\mathbb{F}_{j}, j=0,1$. The second one is a consequence of (i) and the chain rule.

Let $G:=I_{0}(M)$ be the identity component of the group $I(M)$ of $C^{1}$-isometries on $M$. It follows from $\left(\mathrm{A}_{2}\right)$ and from Theorem I.4.6 in [18] that $G$ acts analytically and transitively as a Lie transformation group on $M$, so that $M$ is a homogeneous Riemannian space with respect to $G$. Fix $p_{0} \in M$ and let $H:=\left\{g \in G ; g \cdot p_{0}=p_{0}\right\}$ denote the isotropy group of $p_{0}$, where : $G \times M \rightarrow M$ denotes the action of $G$ on $M$, which is real analytic as well, see again Theorem I.4.6 in [18]. Furthermore, $G / H$ admits a real analytic structure and

$$
j: G / H \rightarrow M, \quad g \cdot H \mapsto g \cdot p_{0}
$$

is a real analytic diffeomorphism, cf. Proposition I.4.2 in [18]. By means of this diffeomorphism we always identify $M$ with the coset manifold $G / H$. Finally, recall that

- $\mathbb{R}^{m}$,

- the unit sphere $\mathbb{S}^{m}=S O(m+1) / S O(m)$,

- products of Riemannian globally symmetric spaces

are Riemannian globally symmetric spaces.

Let $Y$ be a nonempty set. Given $f \in Y^{M}$ and $g \in G$, we define $g \cdot f \in Y^{M}$ by $g \cdot f(p):=f(g \cdot p)$. The next result contains the transformation rule for the operator $Q$ with respect to $G$ which will be needed in the following

Lemma 4.2. Let $g \in G$ be given. Then

(i) $g \cdot \Phi \in \operatorname{Diff}^{\omega}(M, \Sigma)$;

(ii) $g \cdot Q(w, \Phi)=Q(g \cdot w, g \cdot \Phi), \quad w \in D$. 
Proof. (i) This follows from the analyticity of the group action of $G$ on $M$.

(ii) Given $g \in G$ we have

$$
(g \cdot \Phi)^{*} v=g \cdot\left(\Phi^{*} v\right), \quad v \in E_{0}, \quad(g \cdot \Phi)_{*}(g \cdot w)=\Phi_{*} w, \quad w \in F_{0} .
$$

Thus we find

$$
\begin{aligned}
Q(g \cdot w, g \cdot \Phi) & =(g \cdot \Phi)^{*} P\left((g \cdot \Phi)_{*}(g \cdot w)\right)=(g \cdot \Phi)^{*} P\left(\Phi_{*} w\right) \\
& =g \cdot\left(\Phi^{*} P\left(\Phi_{*} w\right)\right)=g \cdot Q(w, \Phi),
\end{aligned}
$$

for any $w \in D$.

We call $G$ a strongly continuous transformation group on the spaces $E_{j}$ if $[v \mapsto$ $g \cdot v] \in \mathcal{L}\left(E_{j}\right)$ for all $g \in G$ and if

$$
a_{v}: G \rightarrow E_{j}, \quad g \mapsto g \cdot v
$$

is continuous at the unit $e$ of $G$ for any $v \in E_{j}$. We next assume that

$G$ is a strongly continuous transformation group on $F_{j}$ for $j=0,1$.

Writing $L(G)$ for the Lie algebra of $G$, we define

$$
T_{X}(t) w:=\exp (t X) \cdot w \quad \text { for } \quad(X, t, w) \in L(G) \times \mathbb{R} \times F_{j} .
$$

It follows from $\left(A_{3}\right)$ that $\left\{T_{X}(t) ; t \in \mathbb{R}\right\}$ is for any $X \in L(G)$ a strongly continuous group on $F_{0}$. We write $A_{X}$ for the infinitesimal generator of $\left\{T_{X}(t) ; t \in \mathbb{R}\right\}$. It follows from Theorem IV.3.3 in [16] that $L(G)$ is finite dimensional. We fix a basis $B=\left\{X_{1}, \ldots, X_{N}\right\}$ of $L(G)$ and assume that

$$
F_{1} \hookrightarrow \operatorname{dom}\left(A_{X}\right) \quad \text { for any } \quad X \in L(G),
$$

where $\operatorname{dom}\left(A_{X}\right)$ is given the graph norm of $A_{X}$. Observe that $\left(A_{4}\right)$ implies that

$$
A_{X} \in \mathcal{L}\left(F_{1}, F_{0}\right) \quad \text { for any } \quad X \in L(G) \text {. }
$$

Recall that, given $v \in F_{j}$, we have set

$$
a_{v}: G \rightarrow F_{j}, \quad g \mapsto g \cdot v
$$

We write $d_{g} a_{v}$ for the differential of $a_{v}$ at $g \in G$, provided $a_{v}$ is differentiable, of course.

Lemma 4.3. (i) If $v \in F_{j}$ then $a_{v} \in C\left(G, F_{j}\right)$ for $j=0,1$.

(ii) If $v \in F_{1}$ then $a_{v} \in C^{1}\left(G, F_{0}\right)$ and, given $(g, X) \in G \times L(G)$, we have

$$
d_{g} a_{v} X=g \cdot A_{X} v
$$


Proof. (i) Let $g \in G$ and choose a sequence $\left(g_{k}\right)$ in $G$ such that $g_{k} \rightarrow g$. Since $G$ is a strongly continuous transformation group on $F_{j}$ we find

$$
a_{v}\left(g_{k}\right)=a_{g \cdot v}\left(g_{k} g^{-1}\right) \rightarrow g \cdot v=a_{v}(g) \text { in } \quad F_{j} .
$$

(ii) Due to (i) and (4.6) it suffices to prove (4.7). Identifying $T_{e} G \cong L(G)$, we have that $\{g \cdot \exp (t X) \quad t \in \mathbb{R}\}$ is an integral curve on $G$ through $g$ with tangent vector $X$. Thus $\left(A_{3}\right)$ yields

$$
\lim _{t \rightarrow 0} \frac{a_{v}(g \cdot \exp (t X))-a_{v}(g)}{t}=g \cdot \lim _{t \rightarrow 0} \frac{\exp (t X) \cdot v-v}{t}=g \cdot A_{X} v \quad \text { in } \quad F_{0},
$$

since $v$ belongs to $F_{1} \subset \operatorname{dom}\left(A_{X}\right)$.

Remark 4.4. Let $M=\Sigma=\mathbb{R}$ and $E_{j}=B U C^{j}(\mathbb{R})$ for $j=0,1$. Then $F_{j}=E_{j}$ for $j=0,1$ and, given $v \in F_{j}$ and $\lambda \in \mathbb{R}$, we have that $a_{v}(\lambda)=\tau_{\lambda} v$, where $\tau_{\lambda} v$ denotes the left translation by $\lambda \in \mathbb{R}$ of $v$. This shows that the regularity of $a_{v}$ with respect to $G$ as stated in Lemma 4.3 is optimal.

Recall that $B=\left\{X_{1}, \ldots, X_{N}\right\}$ denotes a fixed basis of $L(G)$. Given $\left(\mu_{1}, \ldots, \mu_{N}\right\} \in \mathbb{R}^{N}$, we write

$$
\mu B:=\sum_{k=1}^{N} \mu_{k} X_{k}, \quad T_{\mu}(t):=T_{\mu B}(t), \quad A_{\mu}:=A_{\mu B} .
$$

Let $E, F$, and $G$ be Banach spaces. Writing $\mathcal{L}^{2}(E \times F, G)$ for the Banach space of all bilinear continuous mappings from $E \times F$ to $G$, we have

Corollary 4.5. $\left[(\mu, w) \mapsto A_{\mu} w\right] \in \mathcal{L}^{2}\left(\mathbb{R}^{N} \times \mathbb{F}_{1}, \mathbb{F}_{0}\right)$.

Proof. It follows easily from Lemma 4.3 that $\left[(\mu, w) \mapsto A_{\mu} w\right]$ is bilinear. Let $C:=$ $\sum_{k=1}^{N}\left\|A_{X_{k}}\right\|_{\mathcal{L}\left(F_{1}, F_{0}\right)}$ and observe that (4.6) implies that $C<\infty$. Now, given $w \in \mathbb{F}_{1}, \mu \in \mathbb{R}^{N}$, and $t \in I$, we have

$$
\left\|A_{\mu} w(t)\right\|_{F_{0}}=\left\|d_{e} a_{w(t)} \sum_{k=1}^{N} \mu_{k} X_{k}\right\|_{F_{0}} \leq|\mu|_{\mathbb{R}^{N}} \sum_{k=1}^{N}\left\|A_{X_{k}} w(t)\right\|_{F_{0}} \leq C|\mu|\|w\|_{\mathbb{F}_{1}} .
$$

Taking the maximum over $t \in I$, we get the assertion.

We shall now formulate the compatibility condition for the operator $P$ with respect to $G$. For this we first have to introduce the following assumption:

$D$ is invariant under $T_{\mu}(t)$ for all $(\mu, t) \in\left(-\varepsilon_{0}, \varepsilon_{0}\right)^{N} \times I$.

Further, let $\mathbb{D}_{1}:=C(I, D) \cap C^{1}\left(I, F_{0}\right)$, pick $(\mu, w) \in\left(-\varepsilon_{0}, \varepsilon_{0}\right)^{N} \times \mathbb{D}_{1}$, and define

$$
\mathbb{Q}(\mu, w)(t):=Q\left(w(t), T_{\mu}(t) \Phi\right), \quad t \in I,
$$


where $T_{\mu}(t) \Phi:=\exp (t \mu B) \cdot \Phi$ for $(t, \mu) \in \mathbb{R} \times \mathbb{R}^{N}$. Observe that $\left(A_{5}\right)$ ensures that this definition is meaningful. Moreover, we have

$$
\mathbb{Q}(\mu, w)(t)=\left(T_{\mu}(t) \Phi\right)^{*} P\left(\left(T_{\mu}(t) \Phi\right)_{*} w(t)\right), \quad t \in I .
$$

Hence $\mathbb{Q}(\mu, w) \in F_{0}^{I}$. We need that this function belongs to $\mathbb{F}_{0}$ and that the operator $\mathbb{Q}(\mu, w)$ depends analytically on $(\mu, w)$. For this we first observe that

$$
\mathbb{D}_{1} \text { is an open subset of } \mathbb{F}_{1} \text {. }
$$

Indeed, this follows from the compactness of $I$ and the fact that $D$ is open in $F_{1}$. We now assume that there is a $r \in\left(0, \varepsilon_{0}\right)$ such that

$$
[(\mu, w) \mapsto \mathbb{Q}(\mu, w)] \in C^{\omega}\left(\mathbb{B}_{\mathbb{R}^{N}}(0, r) \times \mathbb{D}_{1}, \mathbb{F}_{0}\right) .
$$

Remarks 4.6. a) Assume that $D \rightarrow F_{0}, v \mapsto \Phi^{*} P\left(\Phi_{*} v\right)$ is equivariant with respect to $G$, i.e. $\Phi^{*} P\left(\Phi_{*} g \cdot v\right)=g \cdot \Phi^{*} P\left(\Phi_{*} v\right)$ for $(g, v) \in G \times D$. Then assumption $\left(A_{6}\right)$ is verified. Indeed, recalling $(3.5)$ we get

$$
\begin{aligned}
\mathbb{Q}(\mu, w)(t) & =\left(T_{\mu}(t) \Phi\right)^{*} P\left(\left(T_{\mu}(t) \Phi\right)_{*} w(t)\right) \\
& =T_{\mu}(t) \Phi^{*} P\left(\Phi_{*} T_{\mu}(-t) w(t)\right) \\
& =\Phi^{*} P\left(\Phi_{*} w(t)\right)=Q(w(t), \Phi)
\end{aligned}
$$

for $w \in \mathbb{D}_{1}$ and $t \in I$. Now the assertion follows from (4.2).

b) Assume that $\Sigma=M=\mathbb{R}^{m}$ and that $\Phi=i d$. Furthermore, set

$$
E_{0}:=b u c^{\alpha}\left(\mathbb{R}^{m}\right), \quad B:=E_{1}:=b u c^{2+\alpha}\left(\mathbb{R}^{m}\right)
$$

for some $\alpha \in(0,1)$. We fix $a \in b u c^{\alpha}\left(\mathbb{R}^{m}\right)$ and define

$$
P(v):=a \Delta v \quad \text { for } \quad v \in E_{1} .
$$

Obviously, $P$ is only equivariant under translation if $a$ is constant. In order to satisfy $\left(A_{6}\right)$ for nonconstant coefficient functions $a$, observe that

$$
\mathbb{Q}(\mu, w)(t)=\left(\tau_{t \mu} a\right) \Delta w(t) \quad \text { for } \quad w \in \mathbb{E}_{1},
$$

where $\tau_{t \mu} a$ stands for the left translation of $a$ by the vector $t \mu \in \mathbb{R}^{m}$. Assume now

$$
\begin{aligned}
& a \in B U C^{\infty}\left(\mathbb{R}^{m}\right) \text { and there is a } M_{0}>0 \text { such that } \\
& \left\|\partial^{k} a\right\|_{B U C^{\alpha}\left(\mathbb{R}^{m}\right)} \leq M_{0} k \text { ! for all } k \in \mathbb{N} .
\end{aligned}
$$

Clearly, (4.10) implies that $a \in C^{\omega}\left(\mathbb{R}^{m}\right)$, but observe that (4.10) is in general stronger than pointwise analyticity. If (4.10) holds then $P$ satisfies $\left(A_{6}\right)$. Indeed, given $(\mu, w),(\nu, h) \in$ $\mathbb{R}^{m} \times \mathbb{F}_{1}$ we have

$$
\partial^{k} \mathbb{Q}(\mu, w)[\nu, h]^{k}(t)=\left(\tau_{t \mu} \partial^{k} a\right)[t \nu]^{k} \Delta h(t), \quad t \in I, k \in \mathbb{N},
$$

and therefore

$$
\left\|\partial^{k} \mathbb{Q}(\mu, w)[\nu, h]^{k}\right\|_{\mathbb{F}_{0}} \leq|T \nu|_{\mathbb{R}^{m}}^{k}\|h\|_{\mathbb{F}_{1}}\left\|\partial^{k} a\right\|_{B U C^{\alpha}\left(\mathbb{R}^{m}\right)}, \quad k \in \mathbb{N} .
$$

Recall now that we have by assumption $T \leq 1$. Hence we conclude that $\left(A_{6}\right)$ is satisfied with $r=1$. 
We next fix $\varepsilon_{0}>0$ such that $\lambda t \in\left[0, t^{+}\right)$for all $\lambda \in\left(1-\varepsilon_{0}, 1+\varepsilon_{0}\right)$ and all $t \in[0, T]$. Given now any $(\lambda, \mu) \in\left(1-\varepsilon_{0}, 1+\varepsilon_{0}\right) \times \mathbb{R}^{N}$, we define $v_{\lambda, \mu} \in F_{0}^{I}$ by $v_{\lambda, \mu}(t):=T_{\mu}(t) \Phi^{*} u(\lambda t)$ for $t \in I$.

Our next result shows that the function $v_{\lambda, \mu}$ solves a parameter dependent evolution equation involving the operators $\mathbb{Q}$ and $A_{\mu}$. In order to economize our notation, we set $\Pi:=\Pi\left(\varepsilon_{0}\right):=\left(1-\varepsilon_{0}, 1+\varepsilon_{0}\right) \times\left(-\varepsilon_{0}, \varepsilon_{0}\right)^{N}$.

Lemma 4.7. Given $(\lambda, \mu) \in \Pi$, we have

(i) $v_{\lambda, \mu} \in \mathbb{D}_{1}$.

(ii) $v_{\lambda, \mu}$ solves the evolution equation

$$
\frac{d}{d t} w+\lambda \mathbb{Q}(\mu, w)=A_{\mu} w, \quad w(0)=v_{0},
$$

where $v_{0}:=\Phi^{*} u_{0}$.

Proof. (i) This follows from (2.3), the definition of $D$, see (4.2), assumption $\left(A_{5}\right)$, Lemma 4.3, and the analyticity of the exponential map. Furthermore Lemma 4.3 implies that

$$
\frac{d}{d t} v_{\lambda, \mu}(t)=T_{\mu}(t) A_{\mu}\left(\Phi^{*} u(\lambda t)\right)+\lambda\left(T_{\mu}(t) \Phi\right)^{*} \frac{d}{d t} u(\lambda t), \quad t \in I .
$$

Observing (4.5) and the fact that $T_{\mu}(t)$ and $A_{\mu}$ commute on $F_{1}$ we get

$$
\frac{d}{d t} v_{\lambda, \mu}(t)=A_{\mu} v_{\lambda, \mu}+\lambda\left(T_{\mu}(t) \Phi\right)^{*} \frac{d}{d t} u(\lambda t), \quad t \in I .
$$

(ii) Using (4.13) and (2.4) we now find

$$
\frac{d}{d t} v_{\lambda, \mu}(t)=A_{\mu} v_{\lambda, \mu}-\lambda\left(T_{\mu}(t) \Phi\right)^{*} P(u(\lambda t)), \quad t \in I .
$$

From Lemma 4.3(ii) and the definitions of the operators $Q$ and $\mathbb{Q}$ we further conclude

$$
\begin{aligned}
\left(T_{\mu}(t) \Phi\right)^{*} P\left(\Phi_{*} \Phi^{*} u(\lambda t)\right) & =T_{\mu}(t) Q\left(\Phi^{*} u(\lambda t), \Phi\right) \\
& =Q\left(T_{\mu}(t) \Phi^{*} u(\lambda t), T_{\mu}(t) \Phi\right)=\mathbb{Q}\left(\mu, v_{\lambda, \mu}\right)(t)
\end{aligned}
$$

for $t \in I$. This completes the proof.

Our next lemma contains the key result to show via the implicit function theorem that the mapping $(\lambda, \mu) \mapsto v_{\lambda, \mu}$ is analytic.

Lemma 4.8. Given $((\lambda, \mu), w) \in \Pi \times \mathbb{D}_{1}$, let

$$
F((\lambda, \mu), w):=\left(\frac{d}{d t} w+\lambda \mathbb{Q}(\mu, w)-A_{\mu} w, w(0)-v_{0}\right) .
$$

Then

$$
F \in C^{\omega}\left(\Pi \times \mathbb{D}_{1}, \mathbb{F}_{0} \times F_{1}\right)
$$

and

$$
\partial_{2} F((1,0), w) \in \operatorname{Isom}\left(\mathbb{F}_{1}, \mathbb{F}_{0} \times F_{1}\right), \quad w \in \mathbb{D}_{1},
$$

where $\partial_{2} F$ is the derivative of $F$ with respect to $w \in \mathbb{D}_{1}$. 
Proof. (i) Clearly, we have that

$$
\left(\frac{d}{d t}, \gamma\right) \in \mathcal{L}\left(\mathbb{F}_{1}, \mathbb{F}_{0} \times F_{1}\right)
$$

Hence it follows from Corollary 3.6 that

$$
\left[(\lambda, \mu, w) \mapsto\left(\frac{d}{d t} w-A_{\mu} w, w(0)-v_{0}\right)\right] \in C^{\omega}\left(\mathbb{F}_{1}, \mathbb{F}_{0} \times F_{1}\right) .
$$

Thus we obtain (4.13) from assumption $\left(A_{6}\right)$.

(ii) Let $w \in \mathbb{D}_{1}$ and $h \in \mathbb{F}_{1}$ be given. Then we have

$$
\partial_{2} F((1,0), w) h=\left.\frac{d}{d \varepsilon} F((1,0), w+\varepsilon h)\right|_{\varepsilon=0}=\left(\frac{d}{d t} h+\partial_{1} Q(w, \Phi) h, h(0)\right) .
$$

Combining Lemma 4.2(ii) with Remark III 3.4.2(c) in [2] it follows that, given $(f, \varphi) \in$ $\mathbb{F}_{0} \times F_{1}$, there is a unique solution $h \in \mathbb{F}_{1}$ to the inhomogeneous evolution equation

$$
\frac{d}{d t} h+\partial_{1} Q(w(t), \Phi) h=f(t), \quad h(0)=\varphi
$$

(4.14) is now a consequence of the open mapping theorem.

We are now prepared to show that $v_{\lambda, \mu}$ depends analytically on the parameter $(\lambda, \mu)$.

Proposition 4.9. There is an $\varepsilon_{0}>0$ such that $\left[(\lambda, \mu) \mapsto v_{\lambda, \mu}\right] \in C^{\omega}\left(\Pi\left(\varepsilon_{0}\right), \mathbb{D}_{1}\right)$.

Proof. Let $F$ be given as in Lemma 4.9 and observe that $F((\lambda, \mu), w)=0$ if and only if $w \in \mathbb{D}_{1}$ is a solution to

$$
\frac{d}{d t} w+\lambda \mathbb{Q}(\mu, w)=A_{\mu} w, \quad w(0)=v_{0}
$$

Now the assertion follows from Lemma 4.8, Lemma 4.9, and the implicit function theorem in Banach spaces.

It remains to translate the above Proposition into the desired analyticity of $\hat{u}$, see the beginning of this section.

Theorem 4.10. Assume that $\left(A_{1}\right)-\left(A_{6}\right)$ hold true. Then $\hat{u} \in C^{\omega}\left(\left(0, t^{+}\right) \times \Sigma\right)$.

Proof. (i) Let $\hat{v}:=\Phi^{*} \hat{u}$, i.e. $\hat{v}(t, p):=\hat{u}(t, \Phi(p))$ for $(t, p) \in\left(0, t^{+}\right) \times M$. It suffices to show that $\hat{v} \in C^{\omega}\left(\left(0, t^{+}\right) \times M\right)$. For this we fix $\left(t_{0}, p_{0}\right) \in(0, T) \times M$. Moreover there exists a subset $\left\{j_{1}, \ldots, j_{m}\right\}$ of $\{1, \ldots, N\}$ such that $\left\{X_{j_{1}}, \ldots, X_{j_{m}}\right\} \subset L(G)$ induces via the integral curves $\left[t \mapsto \exp \left(t X_{j_{k}}\right) \cdot p_{0}\right]$ a basis of $T_{p_{0}} M$. Without loss of generality we may assume that $j_{1}=1, \ldots, j_{m}=m$. Moreover, in the following we write $\hat{\mu}=$ $\left(\mu_{1}, \ldots, \mu_{m}, 0, \ldots, 0\right) \in \mathbb{R}^{N}$ for $\left(\mu_{1}, \ldots, \mu_{m}\right) \in \mathbb{R}^{m}$ and we identify

$$
\Pi_{m}:=\Pi_{m}\left(\varepsilon_{0}\right):=\left(1-\varepsilon_{0}, 1+\varepsilon_{0}\right) \times\left(-\varepsilon_{0}, \varepsilon_{0}\right)^{m} \text { with } \Pi \cap\left(\mathbb{R}^{m+1} \times\{0\}\right) .
$$

Shrinking $\varepsilon_{0}>0$ if necessary, we have that

$$
\varphi: \Pi_{m} \rightarrow\left(0, t^{+}\right) \times M, \quad(\lambda, \hat{\mu}) \mapsto\left(\lambda t_{0}, T_{\hat{\mu}}\left(t_{0}\right) \cdot p_{0}\right)
$$


is an analytic parametrization of an open neighborhood $O$ of $\left(t_{0}, p_{0}\right)$ in $\left(0, t^{+}\right) \times M$.

(ii) Observe that by assumption $\left(A_{1}\right)$ we know that $\mathbb{D}_{1} \subset C(I, B U C(M))$. Thus, given $w \in \mathbb{D}_{1}$, the evaluation mapping

$$
\mathbb{D}_{1} \rightarrow \mathbb{R}, \quad w \mapsto w\left(t_{0}\right)\left(p_{0}\right)
$$

is well-defined and clearly analytic. Combining this with Proposition 4.9 we find

$$
\left[(\lambda, \hat{\mu}) \mapsto v_{\lambda, \hat{\mu}}\left(t_{0}\right)\left(p_{0}\right)\right] \in C^{\omega}\left(\Pi_{m}, \mathbb{R}\right) .
$$

But $\varphi^{*} \hat{v}(\lambda, \hat{\mu})=v_{\lambda, \hat{\mu}}\left(t_{0}\right)\left(p_{0}\right)$ for $\lambda, \hat{\mu} \in \Pi_{m}$. This shows that $\hat{v} \in C^{\omega}(O, \mathbb{R})$ and completes the proof.

Proof of Theorem 1.2. (a) Assertion (i) follows from Corollary 3.4.

(b) Setting $E_{j}:=h^{\alpha-1+2 j}(\Sigma)$ for $j=0,1$, hypothesis $\left(A_{1}\right)$ is clearly satisfied.

(c) By assumption there is a isometrically real analytic diffeomorphism $\Phi$ from $\mathbb{S}^{m}$ onto $\Sigma$. Thus hypothesis $\left(A_{2}\right)$ is also satisfied.

(d) It follows from $\left(A_{2}\right)$ that the spaces $F_{j}$ coincide up to an equivalent norm with $h^{\alpha-1+2 j}\left(\mathbb{S}^{m}\right)$ for $j=0$, 1. Clearly, $S O(m+1) \cdot B U C^{\infty}\left(\mathbb{S}^{m}\right) \subset B U C^{\infty}\left(\mathbb{S}^{m}\right)$. Moreover, it is not difficult to verify that $S O(m+1)$ is a strongly continuous transformation group on $B U C^{j}\left(\mathbb{S}^{m}\right)$ for $j=1,2$. By Remark 2.2(c) and a density argument we therefore conclude that $S O(m+1)$ is a strongly continuous transformation group on $h^{1+\alpha}\left(\mathbb{S}^{m}\right)$. Using further the fact that $1-\Delta_{\mathbb{S} m} \in \operatorname{Isom}\left(h^{1+\alpha}\left(\mathbb{S}^{m}\right), h^{\alpha-1}\left(\mathbb{S}^{m}\right)\right)$ and that $\Delta_{\mathbb{S}^{m}}$ is equivariant with respect to $S O(m+1)$ we conclude that $S O(m+1)$ is a strongly continuous transformation group on $h^{\alpha-1}\left(\mathbb{S}^{m}\right)$ as well. This shows that hypothesis $\left(A_{3}\right)$ is satisfied.

(e) Let $X \in L(S O(m+1))$ and $v \in B U C^{2}\left(\mathbb{S}^{m}\right)$ be given. Then it follows from the mean value theorem that

$$
\frac{\exp (t X) \cdot v-v}{t} \rightarrow A_{X} v \quad \text { in } \quad B U C\left(\mathbb{S}^{m}\right) \quad \text { as } \quad t \rightarrow 0
$$

Hence $B U C^{2}\left(\mathbb{S}^{m}\right)$ is contained in $\operatorname{dom}\left(A_{X}\right)$, implying that hypothesis $\left(A_{4}\right)$ is fulfiled.

(f) Let $B:=W(\Sigma)$. Then we have $D=\left\{v \in h^{1+\alpha}\left(\mathbb{S}^{m}\right) ;\|v\|_{B U C^{1+\alpha}}<a / 2\right\}$. Observe further that the metric on $\mathbb{S}^{m}$ is invariant under $S O(m+1)$. This implies that the transformation group on $h^{1+\alpha}\left(\mathbb{S}^{m}\right)$ induced by $S O(m+1)$ consists of isometries, i.e. given $R \in S O(m+1)$, the mapping

$$
h^{1+\alpha}\left(\mathbb{S}^{m}\right) \rightarrow h^{1+\alpha}\left(\mathbb{S}^{m}\right), \quad v \mapsto R \cdot v
$$

is an isometry. This shows that $\left(A_{5}\right)$ is also satisfied.

(g) Finally, fix $v \in D$ and $R \in S O(m+1)$ and set

$$
f:=\theta_{\Phi_{*} v} \circ \Phi \in \operatorname{Diff}^{1+\alpha}\left(\mathbb{S}^{m}, M_{\Phi_{*} v}\right), \quad g:=\theta_{\Phi_{*}(R \cdot v)} \circ \Phi \in \operatorname{Diff}^{1+\alpha}\left(\mathbb{S}^{m}, M_{\Phi_{*}(R \cdot v)}\right) .
$$

Then $R^{*} f=g$ and $R:\left(\mathbb{S}^{m}, g^{*} \eta\right) \rightarrow\left(\mathbb{S}^{m}, f^{*} \eta\right)$ is an isometry, where $\eta$ denotes the Euclidean metric on $\mathbb{R}^{m+1}$. Observe further that $\Phi^{*} K\left(\Phi_{*} v\right)$ and $\Phi^{*} K\left(\Phi_{*}(R \cdot v)\right)$ are, with respect to the ambient space $\left(\mathbb{R}^{m+1}, \eta\right)$, the mean curvatures of $\left(\mathbb{S}^{m}, f^{*} \eta\right)$ and $\left(\mathbb{S}^{m}, g^{*} \eta\right)$, respectively. Thus

$$
R \cdot \Phi^{*} K\left(\Phi_{*} v\right)=\Phi^{*} K\left(\Phi_{*}(R \cdot v)\right) .
$$


Similarly, $\Delta_{\left(\mathbb{S}^{m}, f^{*} \eta\right)}=\Phi^{*} \Delta_{\theta_{\Phi_{*} v}} \Phi_{*}$ and $\Delta_{\left(\mathbb{S}^{m}, g^{*} \eta\right)}=\Phi^{*} \Delta_{\theta_{\Phi_{*}(R \cdot v)}} \Phi_{*}$. Thus we obtain

$$
R \cdot\left(\Phi^{*} \Delta_{\theta_{\Phi_{*} v}} \Phi_{*} w\right)=\Phi^{*} \Delta_{\theta_{\Phi_{*}(R \cdot v)}} \Phi_{*}(R \cdot w), \quad w \in h^{1+\alpha}\left(\mathbb{S}^{m}\right) .
$$

Therefore, given $w \in h^{1+\alpha}\left(\mathbb{S}^{m}\right)$, we also have

$$
R \cdot\left[\Phi^{*}\left(\delta-\mu \Delta_{\theta_{\Phi_{*} v}}\right)^{-1} \Phi_{*} w\right]=\Phi^{*}\left(\delta-\mu \Delta_{\theta_{\Phi_{*}(R \cdot v)}}\right)^{-1} \Phi_{*}(R \cdot w) .
$$

Finally, it follows from the chain rule and $R \cdot f=g$ that

$$
\Phi^{*} L\left(\Phi_{*}(R \cdot v)\right)=R \cdot \Phi^{*} L\left(\Phi_{*} v\right) .
$$

Combining this with (4.15), (4.16), (4.17), we see that

$$
\Phi^{*} P \Phi_{*}: D \rightarrow h^{\alpha-1}\left(\mathbb{S}^{m}\right), \quad v \mapsto \Phi^{*}\left[L\left(\Phi_{*} v\right) \Delta_{\Phi_{*} v}\left(\delta-\mu \Delta_{\Phi_{*} v}\right)^{-1} K\left(\Phi_{*} v\right)\right]
$$

is equivariant with respect to $S O(m+1)$. Now the the second assertion follows from Theorem 4.10 .

\section{References}

[1] Amann H, Nonhomogeneous Linear and Quasilinear Elliptic and Parabolic Boundary Value Problems, in Function Spaces, Differential Operators and Nonlinear Analysis, Editors: Schmeisser H J and Triebel H, Teubner, Stuttgart, Leipzig, 1993, 9-126.

[2] Amann H, Linear and Quasilinear Parabolic Problems, Vol. I, Birkhäuser, Basel, 1995.

[3] Amann H, Elliptic Operators with Infinite-Dimensional State Spaces, J. Evol. Equs. 1 (2001), $143-188$.

[4] Angenent S, Nonlinear Analytic Semiflows, Proc. Roy. Soc. Edinburgh 115A (1990), 91-107.

[5] Aubin T, Nonlinear Analysis on Manifolds. Monge-Ampére Equations, Springer, New York, 1992.

[6] Baillon J B, Caractère borné de certains générateurs de semigroup linéaire dans les espaces de Banach. C.R. Acad. Sc. Paris 290A (1980), 757-760.

[7] Da Prato G and Grisvard P, Equations d'évolution abstraites nonlinéaires de type parabolique. Ann. Mat. Pura Appl. 120 (1979), 329-396.

[8] Cahn J W and Taylor J E, Surface Motion by Surface Diffusion, Acta Metallurgica 42 (1994), $1045-1063$.

[9] Elliott C M and Garcke H, Existence Results for Diffusive Surface Motion Laws, Adv. Math. Sci. Appl. 7 (1997), 467-490.

[10] Escher J, Giga Y and Ito K, On a Limiting Motion and Self-Intersection for the Intermediate Surface Diffusion Flow, J. Evol. Equs. 2 (2002), 349-364.

[11] Escher J and Simonett G, Classical Solutions of Multidimensional Hele-Shaw Models, SIAM J. Math. Anal. 28 (1997), 1028-1047. 
[12] Escher J and Simonett G, Classical Solutions for the Hele-Shaw Models with Surface Tension, Adv. Diff. Equs. 2 (1997), 619-642.

[13] Escher J, Mayer U F and Simonett G, The Surface Diffusion Flow for Immersed Hypersurfaces, SIAM J. Math. Anal. 29 (1999), 1419-1433.

[14] Escher J and Simonett G, Moving Surfaces and Abstract Parabolic Evolution Equations, Progress in Nonlinear Differential Equations and Their Applications 35 (1999), 183-212.

[15] Escher J and Simonett G, Analyticity of Solutions to Fully Nonlinear Parabolic Evolution Equations on Symmetric Spaces, J. Evol. Equs., to appear.

[16] Helgason S, Differential Geometry and Symmetric Spaces, Academic Press, New York, 1962.

[17] Klingenberg W, Riemannian Geometry, de Gruyter, Berlin 1982.

[18] Kobayashi S and Nomizu K, Foundations of Differential Geometry, Wiley, New York, 1963.

[19] Lunardi A, Analytic Semigroups and Optimal Regularity in Parabolic Equations. Birkhäuser, Basel, 1991.

[20] Seeley R T, Norms and Domains of the Complex Powers Az, Amer. J. Math. 93 (1971), 299-309.

[21] Simonett G, Center Manifolds for Quasilinear Reaction-Diffusion Systems, Differential Integral Equations 8 (1995), 753-796.

[22] Taylor J E and Cahn J W, Linking Anisotropic Sharp and Diffuse Surface Motion Laws via Gradient Flow, J. Stat. Phys. 77 (1994), 183-197.

[23] Triebel H, Theory of Function Spaces, Birkhäuser, Basel, 1983.

[24] Triebel H, Theory of Function Spaces II, Birkhäuser, Basel, 1992.

[25] Yosida K, Functional Analysis, Springer, Berlin, 1980. 